\title{
Investigation and Suppression of Fiber Nonlinearities Using Injection-Locking in OFDM-WDM System
}

\author{
Monika Nehra (iD) and Deepak Kedia $(1)$ \\ Department of Electronics and Communication Engineering, Guru Jambheshwar University of Science \& Technology, \\ Hisar, Haryana 125001, India \\ Correspondence should be addressed to Deepak Kedia; kedia29@gmail.com
}

Received 30 June 2018; Accepted 24 September 2018; Published 1 November 2018

Academic Editor: Sulaiman W. Harun

Copyright (c) 2018 Monika Nehra and Deepak Kedia. This is an open access article distributed under the Creative Commons Attribution License, which permits unrestricted use, distribution, and reproduction in any medium, provided the original work is properly cited.

\begin{abstract}
The fiber nonlinearities play a major role in optical communication with respect to the system performance and transmission capacity. Here, fiber nonlinear impairments are investigated through consideration of several parameters of optical fiber system, such as fiber length and core effective area. We also demonstrate the mitigation of fiber nonlinearities by using single-stage injectionlocking in $3 \times 10 \mathrm{~Gb} / \mathrm{s}$ OFDM-WDM system. This paper focuses on selection of appropriate bias current for slave laser to suppress the four wave mixing (FWM) crosstalk effects. These findings resemble the importance of single-stage injection-locking for FWM suppression for high-speed data communication.
\end{abstract}

\section{Introduction}

Over the time, the requirements for bandwidth have been increased critically. The different possible solutions have come up which include architectural modifications in terms of modulator design, advance modulation formats, and integration of wireless communication with optical fiber technologies. However, there are several issues that need to be resolved such as fiber dispersion, noise, and fiber nonlinearities. With increased power levels, the different fiber nonlinearities come into picture such as four wave mixing (FWM), self-phase modulation (SPM), cross-phase modulation (CPM), stimulated Raman scattering (SRS), and stimulated Brillouin scattering (SBS). Out of these effects, the FWM and SBS processes have significant potential to alter the performance of transmission systems, even if the systems are operated using narrow linewidth single frequency lasers $[1,2]$. These effects become more critical to handle in higherchannel WDM system for long distance transmission. The FWM products result throughout the changes in refractive index of fiber core with respect to optical intensity. If the FWM products fall in transmission window of baseband signals, these can give rise to crosstalk between significant channels propagating through the fiber [3]. These effects are analogous to intermodulation distortion in case of electrical systems.

The efficiency of FWM products generally depends on the fiber-chromatic dispersion, transmission length, and the channel separation. Therefore, diverse research efforts have been reported to mitigate the crosstalk due to FWM in appropriate transmission system design [4-7]. Guo and Shu [8] reported the suppression of FWM crosstalk using Raman assisted fiber optical parametric amplifier (FOPA). The Raman assisted FOPA system offered unique power evolution of signals that helped obtain $7 \mathrm{~dB}$ decrease in FWM crosstalk. Zhu et al. [9] reported microwave signals generation based on a novel technique utilizing optical sideband injection and FWM. This technique can produce microwave signals with frequency octupling, 12- and 14tupling. However, the several limitations can be seen in terms of bandwidth limited photodetector that allows the signal generation up to $18 \mathrm{GHz}$ only. Recently, injectionlocking has emerged as one of the most promising techniques for suppression of fiber nonlinearities. Jignesh et al. [10] proposed the injection-locking technique to compensate the interchannel nonlinear phase noise that occurs mainly due to XPM. The injection-locking strongly follows the XPM-phase distortion that can be partially cancelled with the help of 
homodyne receiver. No doubt injection-locking technique is very effective for microwave signal generation or XPM-phase noise cancellation; it can outperform other techniques in the suppression of other fiber nonlinearities also.

Here, the fiber length and core effective area are investigated in terms of their effects on FWM power. The suppression of FWM products using master-slave injection-locking is a new concept and not much explored. The selection of bias current for slave laser has a significant impact over the system performance in terms of suppression of FWM products.

\section{Concept of Fiber Nonlinearities}

The terms linear and nonlinear optics basically relate to intensity-independent and intensity-depenedent phenomenon, respectively. The linear impairments usually originate due to chromatic dispersion (CD), fiber loss, and polarization mode dispersion (PMD). On the other hand, the nonlinear impairments are caused by two different mechanisms: (i) inelastic scattering of photons inside the fiber (e.g., SBS and SRS nonlinearities) and (ii) changes in the refractive index of fiber core via changes in optical power intensity (e.g., FWM, SPM, and XPM nonlinearities) [11]. Out of these nonlinear effects, FWM is a third-order nonlinearity that comes into picture when two or more than two signals are propagated through the fiber. The number of FWM crosstalks increases rapidly with the number of propagated signals $(N)$ as $(1 / 2) N^{3}(N-1)$ [12]. The power level of FWM products is strongly dependent on channel spacing, fiber dispersion, and core effective area. The magnitude of FWM can be expressed $[13,14]$ as

$$
P_{F W M}=\frac{\eta}{9} D^{2} \gamma^{2} P_{i} P_{j} P_{k} e^{(-a L)}\left\{\frac{\left[1-e^{(-a L)}\right]^{2}}{a^{2}}\right\},
$$

where $\eta$ denotes the FWM efficiency, D denotes the CD of the fiber, $P_{i}, P_{j}$, and $P_{k}$ denote input optical power with frequency $f_{i}, f_{j}$, and $f_{k}$, respectively (given that $k \neq i$ ), L denotes the fiber length, $\alpha$ denotes the attenuation coefficient, and $\gamma$ is the nonlinear coefficient of the fiber, given as

$$
\gamma=\frac{2 \pi \mathrm{n}_{2}}{\lambda \mathrm{A}_{\mathrm{eff}}}
$$

where $n_{2}$ is the nonlinear refractive index of optical fiber, $\lambda$ is the operating wavelength of the fiber, and $A_{e f f}$ is the core effective area. The FWM efficiency can be expressed [15] as

$$
\eta=\frac{\alpha^{2}}{\alpha^{2}+\beta^{2}}\left[1+\frac{4 \exp (-\alpha L) \sin ^{2}(\Delta \beta L / 2)}{\left.(1-\exp (-\alpha L))^{2}\right)}\right],
$$

where $\Delta \beta$ is the phase-matching factor and can be expressed as

$$
\begin{aligned}
\Delta \beta= & \beta-\beta_{o} \\
= & 2 \pi\left(f-f_{o}\right) \frac{d \beta}{d \omega}+\frac{1}{2} 2 \pi\left(f-f_{o}\right)^{2} \frac{d^{2} \beta}{d \omega^{2}} \\
& +\frac{1}{6} 2 \pi\left(f-f_{o}\right)^{3} \frac{d^{3} \beta}{d \omega^{3}}+\frac{1}{24} 2 \pi\left(f-f_{o}\right)^{4} \frac{d^{4} \beta}{d \omega^{4}} \\
& +\frac{1}{120} 2 \pi\left(f-f_{o}\right)^{5} \frac{d^{5} \beta}{d \omega^{5}} .
\end{aligned}
$$

Here, dispersion parameters are considered up to fifth order. The phase-matching factor varies along with the wave propagation through the fiber such as

$$
\Delta \beta^{\prime}=\Delta \beta-\gamma m\left(P_{1} P_{2} P_{3}\right)\left[\frac{1-x p\left(-\alpha L_{e f f}\right)}{\alpha L_{e f f}}\right],
$$

where $\mathrm{m}$ is an integer and $L_{e f f}$ is the effective fiber length, i.e., $L \gg L_{e f f}$. Further, the effect of different parameters of optical fiber on FWM crosstalk is investigated in the next section.

\section{Investigation of FWM Effects}

In order to investigate the effect of FWM-induced crosstalk, the powers of all input channels are considered equal. The other parameters are considered as per the ITU:T recommendation G.653 as fiber CD $(D)$ is $0.5 \mathrm{ps} / \mathrm{km} \mathrm{nm}$ along with $0.25 \mathrm{~dB} / \mathrm{km}$ attenuation factor $(\alpha)$. The dispersion effect is considered up to 5 th order with $m=0.63$. The effective core area is $67.43 \mu \mathrm{m}^{2}$ and fiber nonrefractive index $\left(n_{2}\right)$ is chosen as $2.68 \times 10^{-20} \mathrm{~m}^{2} / \mathrm{W}$. Figure 1 shows the FWM crosstalk induced in optical fiber system due to combined effect of dispersion up to fifth order. As the fiber length increases, the FWM power products become more critical to handle (refer to Figure 1(a)). Similarly, fiber effective area also puts limitation on its further decrement beyond $50 \mu \mathrm{m}^{2}$ that can give rise to higher FWM nonlinearities (refer to Figure 1(b)).

\section{Suppression of FWM Effects using Injection-Locking}

As already discussed in Section 3, the optical transmission power, channel length, and the core effective area put several limitations on the system in terms of their careful selection in order to suppress the fiber nonlinearities, i.e., FWM. The FWM effects occur due to confinement of optical field to the small effective area of fiber core over long distances. However, the core effective area as well as the optical transmission power and/or channel length cannot be increased and decreased respectively beyond certain limits. Therefore, we have put efforts over the suppression of FWM products by laser injection-locking technique. The injectionlocking can suppress the FWM products by deviating the spacing between master and slave operating wavelengths [16]. The simulation setup for 3-channel OFDM-WDM system is shown in Figure 2. The generation of OFDM signals is presented elsewhere [17]. In brief, OFDM signal makes use of $N$ closely spaced orthogonal subcarriers that are transmitted in parallel configuration. The advanced modulation technique (i.e., 16-quadrature amplitude modulation) is used to separately modulate the subcarriers. In the OFDM technique, guard band is also provided at the end of each OFDM stream in order to reduce the intersymbol interference (ISI). The three OFDM signals are generated and separately modulated with the help of Mach-Zehnder modulator (MZM) at different wavelengths. The MZM can generate optical combs with excellent properties in terms of spectral flatness. The MZM can also cause nonlinear effects depending on its static phase 


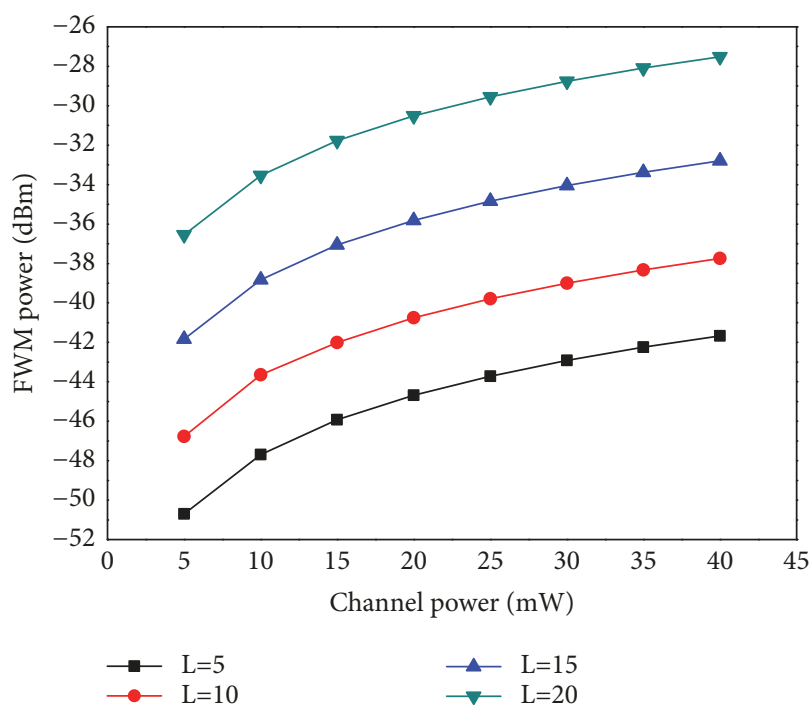

(a)

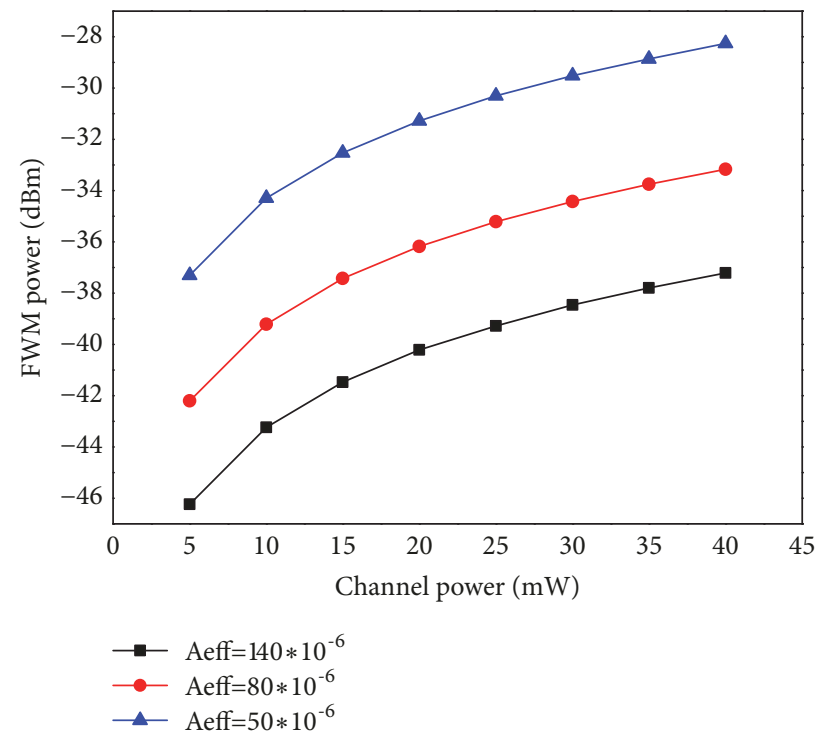

(b)

FIGURE 1: Dependence of FWM crosstalk products over channel power at different values: (a) fiber length (L) and (b) core effective area $\left(A_{e f f}\right)$.

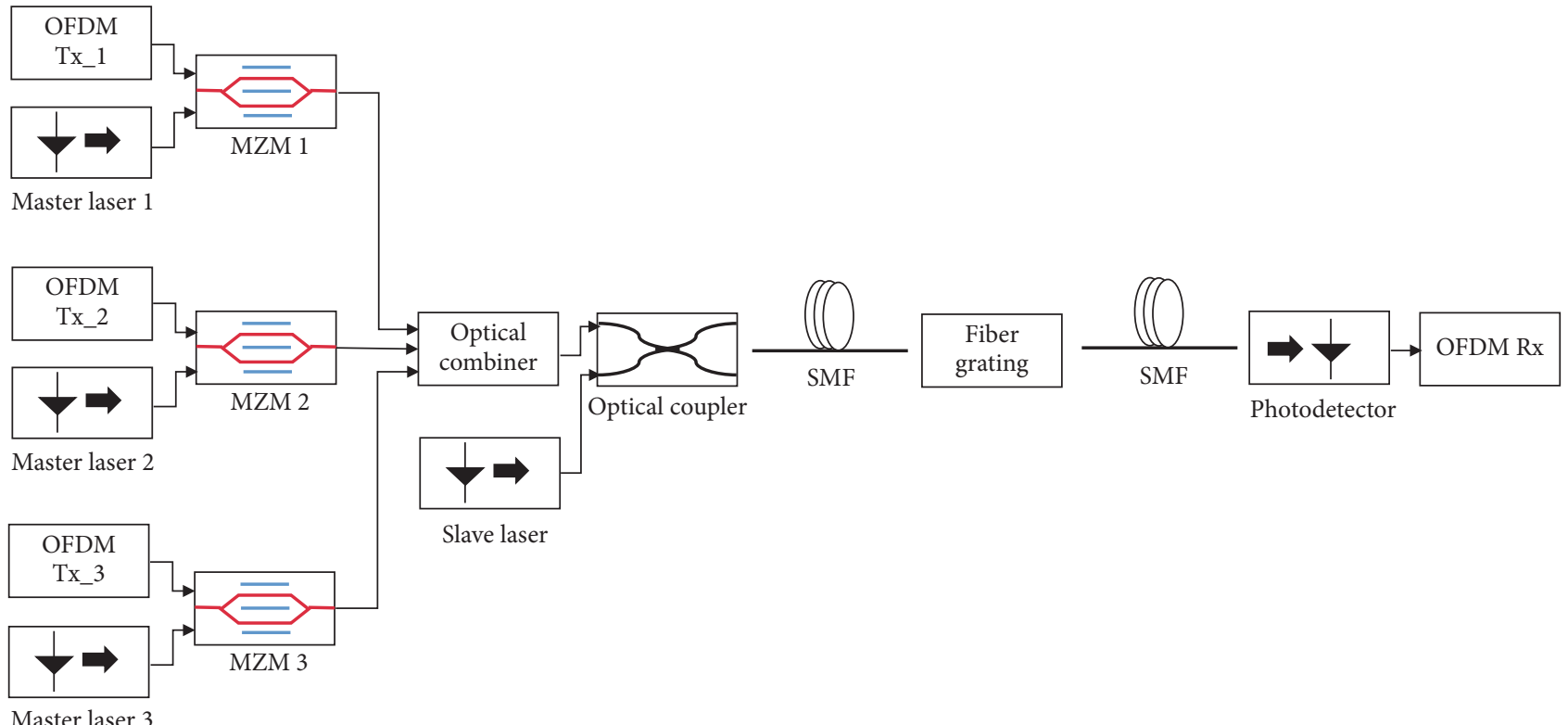

FIGURE 2: Configuration of 3-channel OFDM-WDM system using master-to-slave injection-locking technique.

shift or bias point. The power transfer characteristics of MZM are good at quadrature bias point for direct-detection systems [18].

The different parameters of OFDM-WDM system are listed in Table 1. Further, to suppress the effect of FWM crosstalk, injection-locking technique is employed. In the proposed technique, we have derived the slave laser at a nearby frequency of master lasers. This injection ratio can be defined as $P_{\text {master }} / P_{\text {slave }}$, where $P_{\text {master }}$ and $P_{\text {slave }}$ are the optical powers in case of master and slave laser, respectively. A comparative analysis of FWM crosstalk generation in optical system is presented in Figure 3. The injection-locking technique offers better performance by lowering the magnitude of FWM products, i.e. $\sim 20 \mathrm{~dB}$ reduction in power level of FWM crosstalk products, than that of without injection-locking.

Further, the locking conditions of both (master and slave) lasers affect the system performance. In case of unstable injection-locking, the strong oscillations may appear in the output power caused due to beating in between the components of the optical field. The output power of injection-locking system depends upon the frequency detuning between the free-running slave laser and the injected 


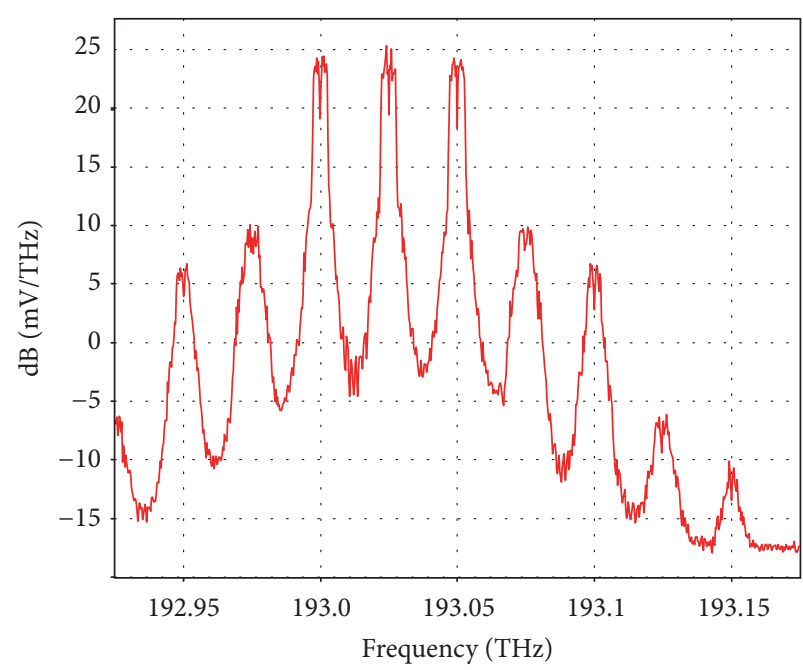

(a)

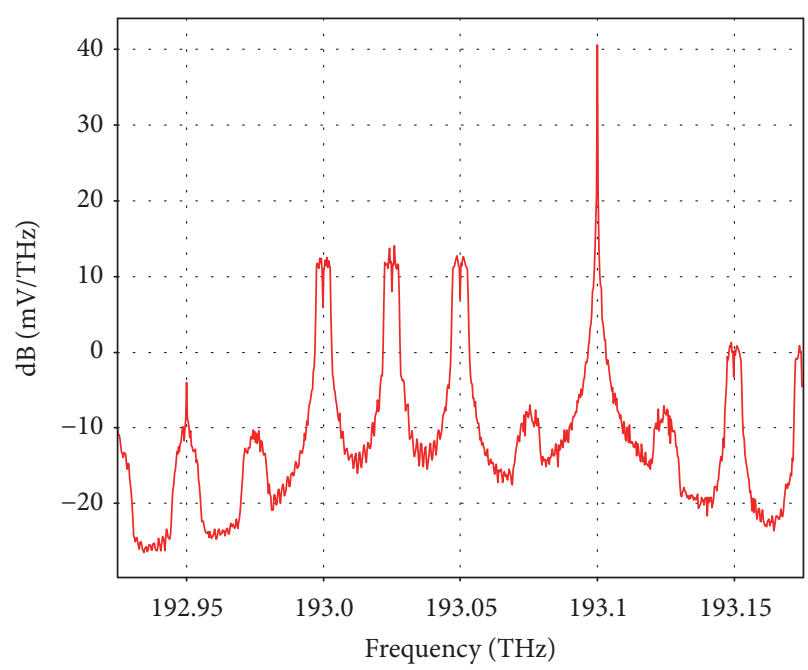

(b)

FIGURE 3: Received optical spectra of OFDM-WDM system (a) without injection-locking and (b) with injection-locking.

TABLE 1: Different parameters of 3-channel OFDM-WDM system.

\begin{tabular}{lc}
\hline Parameters & Value \\
\hline Data rate & $10 \mathrm{~Gb} / \mathrm{s}$ \\
OFDM subcarriers & 64 \\
Operating wavelength for master laser 1 & $1553.33 \mathrm{~nm}$ \\
Operating wavelength for master laser 2 & $1553.13 \mathrm{~nm}$ \\
Operating wavelength for master laser 3 & $1552.93 \mathrm{~nm}$ \\
Operating wavelength for slave laser & $1552.53 \mathrm{~nm}$ \\
Channel spacing & $25 \mathrm{GHz}$ \\
Fiber length & $2 \times 100 \mathrm{~km}$ \\
MZM bias point & $\pi / 2$ \\
\hline
\end{tabular}

optical beam. The biasing of semiconducting laser in such optical injection can help in locking of optical field components with respect to the current modulation and the optical injection [19]. The bias point should be carefully chosen in order to obtain perfect locking conditions. If the slave laser power is not strong enough to affect the locking conditions, the optical frequencies may operate in FWM regime. Figure 4 shows the variation in distribution of power among FWM cross-products as function of bias current for slave laser. The bias current of slave laser is varied from $8 \mathrm{~mA}$ to $40 \mathrm{~mA}$. At high injection ratio, the FWM crosstalk occurs with high power levels and can degrade the system performance. Therefore, without increasing the optical power levels of master lasers, the strength of optical signal from slave laser can be tuned with the help of bias currents [20]. As biasing of slave laser increases from $8 \mathrm{~mA}$ to $40 \mathrm{~mA}$, the power level of FWM products decreases significantly (refer to Figures 4(a)-4(f)). However, further increment in bias current of slave laser does not cause any significant reduction in power level of FWM products (refer to Figures $4(\mathrm{~g})-4(\mathrm{~h}))$. These variations in power of FWM products near the operating wavelengths of master(s) and slave lasers are plotted in Figure 5. The appropriate dc-biasing of slave laser offers improved modulation characteristics for better locking conditions in order to suppress the FWM products.

From Figure 5, it is clear that a threshold point has been achieved for appropriate biasing of slave laser in order to suppress the FWM products, i.e., $40 \mathrm{~mA}$. The comparative analysis of injection-locking technique is also carried out with respect to existing literature (refer to Table 2).

Therefore, injection-locking technique offers effective suppression of FWM effects and can enhance the system performance by avoiding the complexity of the system.

\section{Conclusion}

In this paper, we have investigated the fiber nonlinearities, especially FWM. There are several parameters that may contribute to reducing the power level of FWM crosstalk products, i.e., appropriate selection of channel spacing and core effective area. However, significant decrement in FWM crosstalk power levels can be obtained by employing the injection-locking technique, i.e., $\sim 20 \mathrm{~dB}$ reduction in power level of FWM crosstalk products. The slave laser should be biased appropriately in order to obtain stable-locking conditions. We have achieved excellent suppression of FWM products at a bias current of $40 \mathrm{~mA}$ for slave laser.

\section{Data Availability}

The data used to support the findings of this study are available from the corresponding author upon request.

\section{Conflicts of Interest}

The authors declare that there are no conflicts of interest regarding the publication of this paper. 
TABLE 2: Critical analysis of the proposed technique with respect to existing literature.

\begin{tabular}{|c|c|c|}
\hline Research work(s) & Proposed mechanism & Outcomes \\
\hline Bordonalli et al., 2015 & Injection-locking technique & $\begin{array}{l}\text { Optical comb generation with reduced phase } \\
\text { noise spectral density (below }-110 \mathrm{dBc} / \mathrm{Hz} \text { ) }\end{array}$ \\
\hline Zhu et al., 2017 & $\begin{array}{l}\text { Combined effect of FWM and } \\
\text { injection-locking }\end{array}$ & Photonic generation of microwave signals \\
\hline Jignesh et al., 2018 & Injection-locking technique & XPM-phase distortion cancellation \\
\hline Hu et al., 2017 & Multiple optical phase conjugation pairs & Fiber nonlinearity mitigation \\
\hline Proposed work & Single-stage injection-locking technique & $\begin{array}{l}\text { Suppression }(\sim 20 \mathrm{~dB}) \text { of power level of FWM } \\
\text { crosstalk }\end{array}$ \\
\hline
\end{tabular}

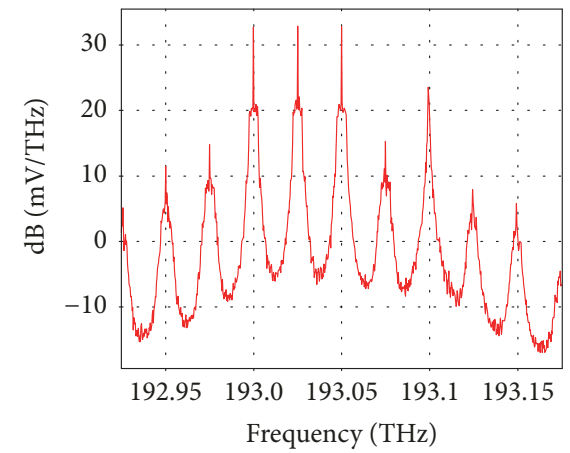

(a)

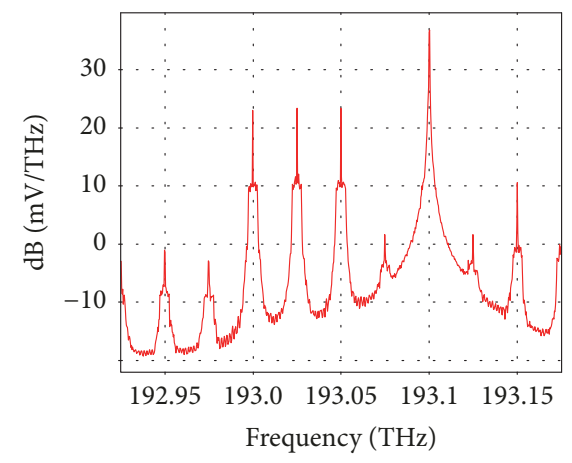

(d)

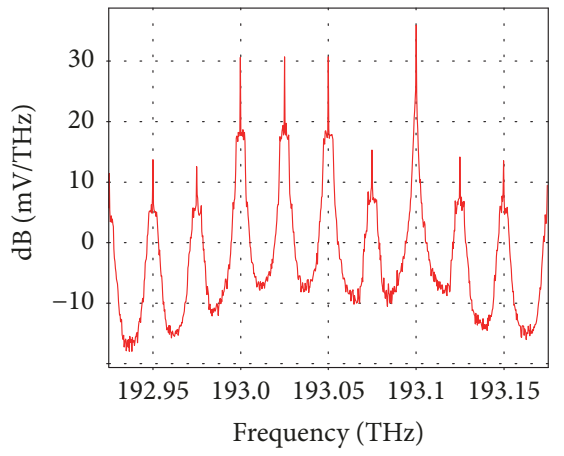

(b)

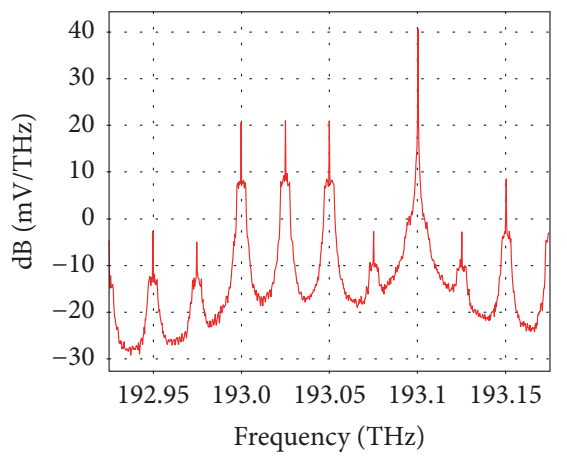

(e)

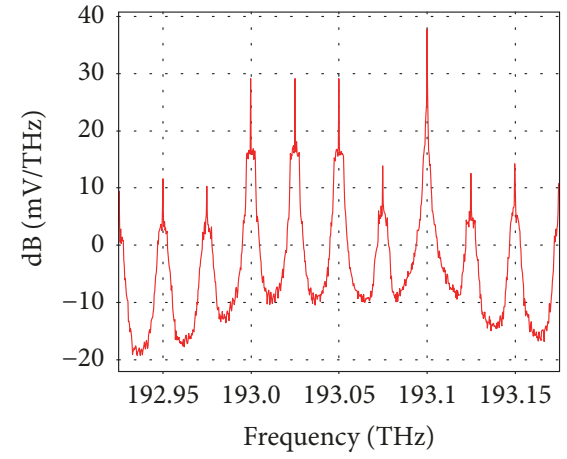

(c)

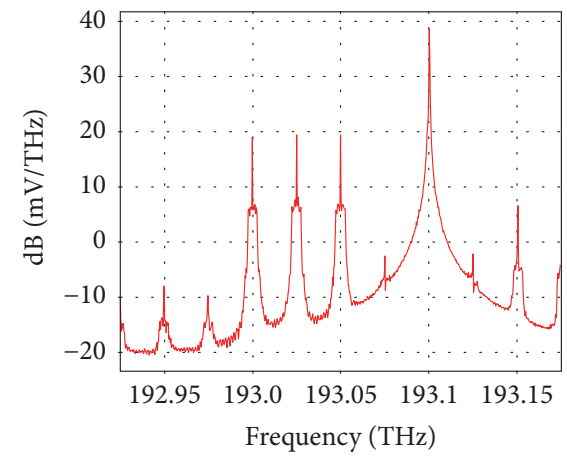

(f)

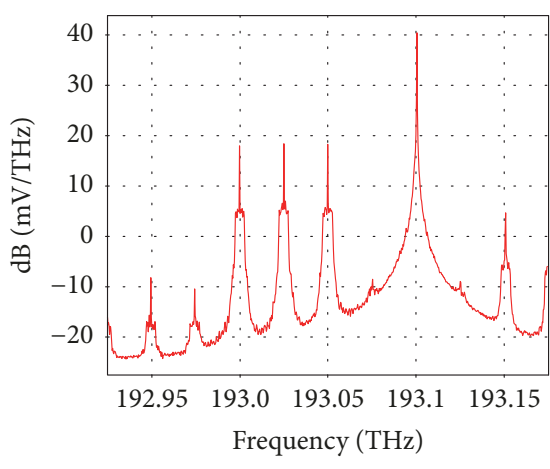

(g)

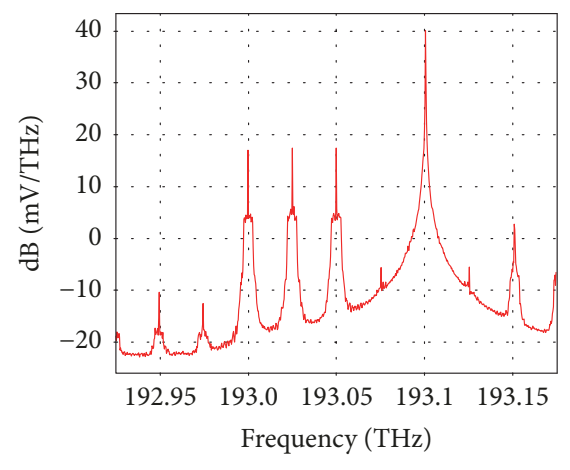

(h)

FIGURE 4: Received optical spectra with significant FWM crosstalk products as a function of bias current of slave laser: (a) $8 \mathrm{~mA}$, (b) $9 \mathrm{~mA}$, (c) $10 \mathrm{~mA}$, (d) $20 \mathrm{~mA}$, (e) $30 \mathrm{~mA}$, (f) $40 \mathrm{~mA}$, (g) $50 \mathrm{~mA}$, and (h) $60 \mathrm{~mA}$. 


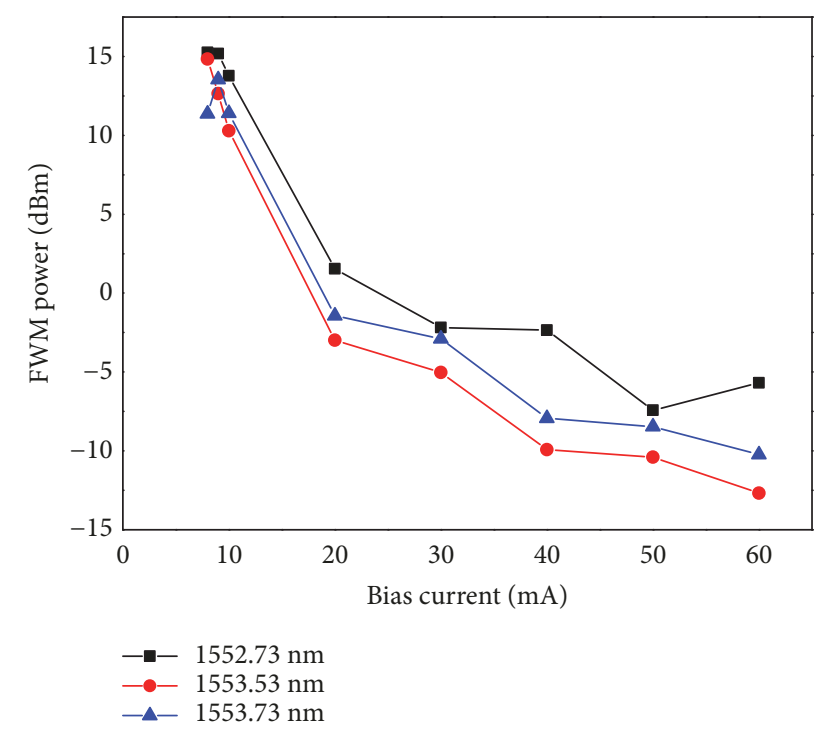

FIGURE 5: Optical power-to-bias current curves for FWM products near the operating wavelengths of master(s) and slave laser.

\section{Acknowledgments}

Monika Nehra thanks the UGC, India, for providing financial assistance in the form of JRF (Award no. 3608 dated 29-022016).

\section{References}

[1] S. Sugavanam, N. Tarasov, X. Shu, and D. V. Churkin, "Narrowband generation in random distributed feedback fiber laser," Optics Express, vol. 21, no. 14, pp. 16466-16472, 2013.

[2] A. Sincore, N. Bodnar, J. Bradford, A. Abdulfattah, L. Shah, and M. C. Richardson, "SBS Threshold Dependence on Pulse Duration in a $2053 \mathrm{~nm}$ Single-Mode Fiber Amplifier," Journal of Lightwave Technology, vol. 35, no. 18, pp. 4000-4003, 2017.

[3] D. E. Ceballos-Herrera, R. Gutierrez-Castrejon, and J. A. Alvarez-Chavez, "Stimulated Raman scattering and four-wave mixing effects on crosstalk of multicore fibers," IEEE Photonics Technology Letters, vol. 30, pp. 63-66, 2018.

[4] E. Temprana, E. Myslivets, B. P.-P. Kuo et al., "Overcoming Kerrinduced capacity limit in optical fiber transmission," Science, vol. 348, no. 6242, pp. 1445-1448, 2015.

[5] J. Kaur and H. Singh, "Placement of optical phase conjugation in WDM systems to suppress FWM," Microwave and Optical Technology Letters, vol. 60, no. 3, pp. 704-708, 2018.

[6] M. Nehra and D. Kedia, "Design of Optical I/Q Modulator Using Dual-drive Mach-Zehnder Modulators in Coherent Optical-OFDM System," Journal of Optical Communications, vol. 39, no. 2, pp. 155-159, 2018.

[7] X. Guo and C. Shu, "Investigation of raman-assisted crosstalk reduction in multi-wavelength fiber optical parametric amplification," Journal of Lightwave Technology, vol. 33, no. 23, pp. 4746-4751, 2015.

[8] H. Hu, R. M. Jopson, A. H. Gnauck, S. Randel, and S. Chandrasekhar, "Fiber nonlinearity mitigation of WDM-PDM QPSK/16-QAM signals using fiber-optic parametric amplifiers based multiple optical phase conjugations," Optics Express, vol. 25, no. 3, pp. 1618-1628, 2017.
[9] H. Zhu, R. Wang, P. Xiang et al., "A novel approach to photonic generate microwave signals based on optical injection locking and four-wave mixing," Optics Communications, vol. 400, pp. 101-105, 2017.

[10] J. Jignesh, A. Lowery, and B. Corcoran, "Inter-channel nonlinear phase noise compensation using optical injection locking," Optics Express, vol. 26, no. 5, pp. 5733-5746, 2018.

[11] M. P. Yankov, K. J. Larsen, and S. Forchhammer, “Temporal probabilistic shaping for mitigation of nonlinearities in optical fiber systems," Journal of Lightwave Technology, vol. 35, no. 10, pp. 1803-1810, 2017.

[12] S. T. Le, K. Blow, and S. Turitsyn, "Power pre-emphasis for suppression of FWM in coherent optical OFDM transmission," Optics Express, vol. 22, no. 6, pp. 7238-7248, 2014.

[13] D. Uzunidis, C. Matrakidis, and A. Stavdas, "An improved model for estimating the impact of FWM in coherent optical systems," Optics Communications, vol. 378, pp. 22-27, 2016.

[14] K. Inoue and H. Toba, "Fiber Four-Wave Mixing in MultiAmplifier Systems with Nonuniform Chromatic Dispersion," Journal of Lightwave Technology, vol. 13, no. 1, pp. 88-93, 1995.

[15] T. Yamamoto and M. Nakazawa, "Highly efficient four-wave mixing in an optical fiber with intensity dependent phase matching," IEEE Photonics Technology Letters, vol. 9, no. 3, pp. 327-329, 1997.

[16] H.-Y. Chen, Y.-C. Chi, C.-Y. Lin, C.-T. Tsai, and G.-R. Lin, "Four-wave-mixing suppression of master-to-slave injectionlocked two-wavelength FPLD Pair for MMW-PON," Journal of Lightwave Technology, vol. 34, no. 20, pp. 4810-4818, 2016.

[17] D. Kedia and M. Nehra, "Extended Reach 10 Gb/s Transmission with an Optical I/Q Modulator Using VCSELs over OFDMBased Multimode Fiber Link," International Journal of Optics, vol. 2018, Article ID 2395495, 7 pages, 2018.

[18] Y. Tang, W. Shieh, X. Yi, and R. Evans, "Optimum design for RF-to-optical up-converter in coherent optical OFDM systems," IEEE Photonics Technology Letters, vol. 19, no. 7, pp. 483-485, 2007.

[19] T. B. Simpson and F. Doft, "Double-locked laser diode for microwave photonics applications," IEEE Photonics Technology Letters, vol. 11, no. 11, pp. 1476-1478, 1999.

[20] J. Al Roumy, J. Perchoux, Y. L. Lim, T. Taimre, A. D. Rakic, and T. Bosch, "Effect of injection current and temperature on signal strength in a laser diode optical feedback interferometer," Applied Optics, vol. 54, no. 2, pp. 312-318, 2015. 

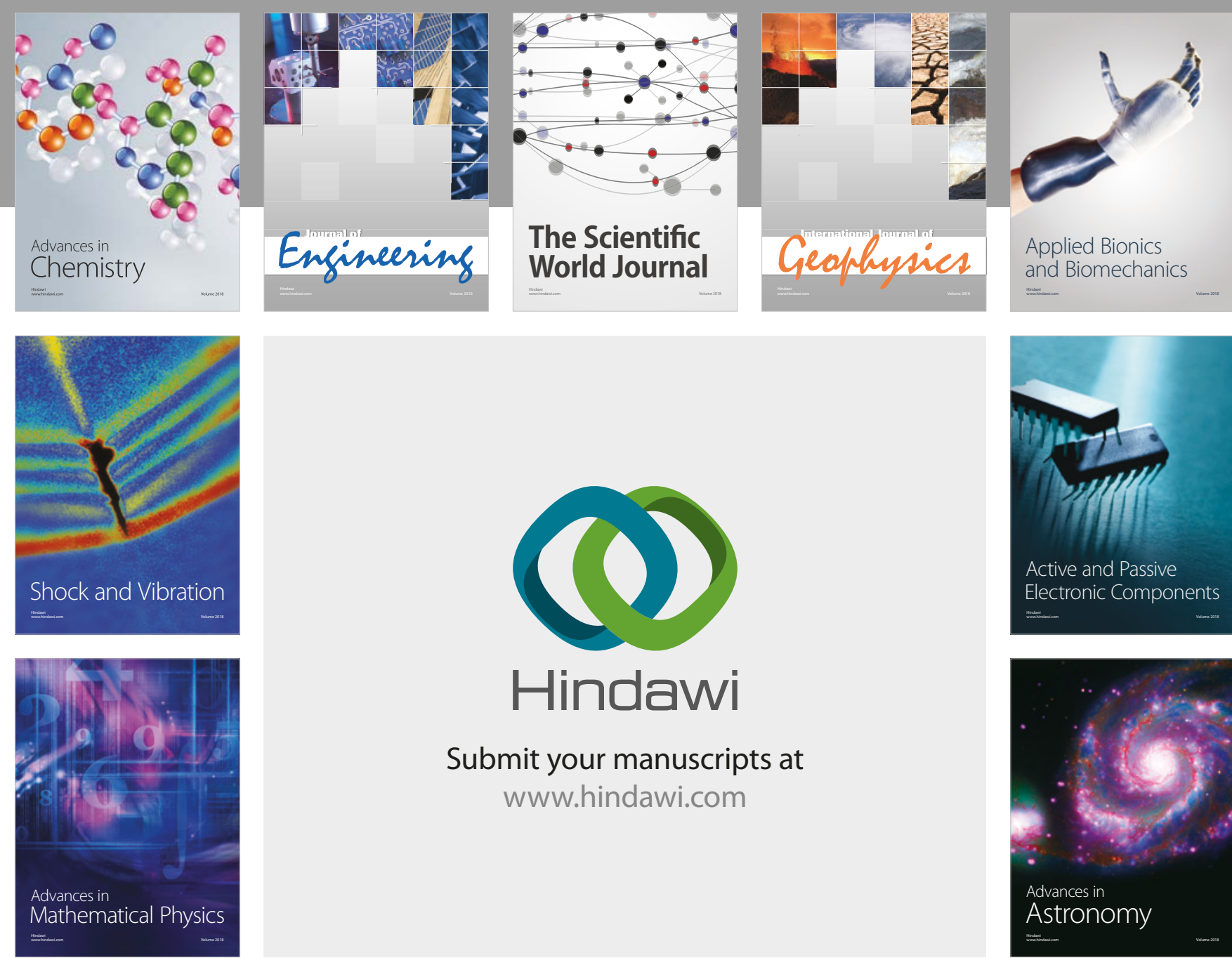

Submit your manuscripts at

www.hindawi.com

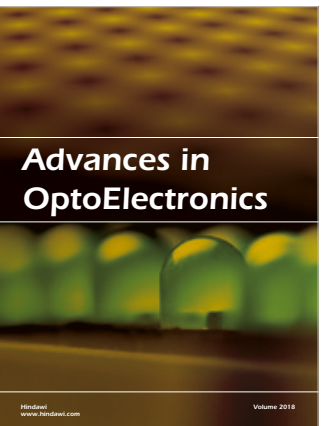

\section{Rotcting Machinery}
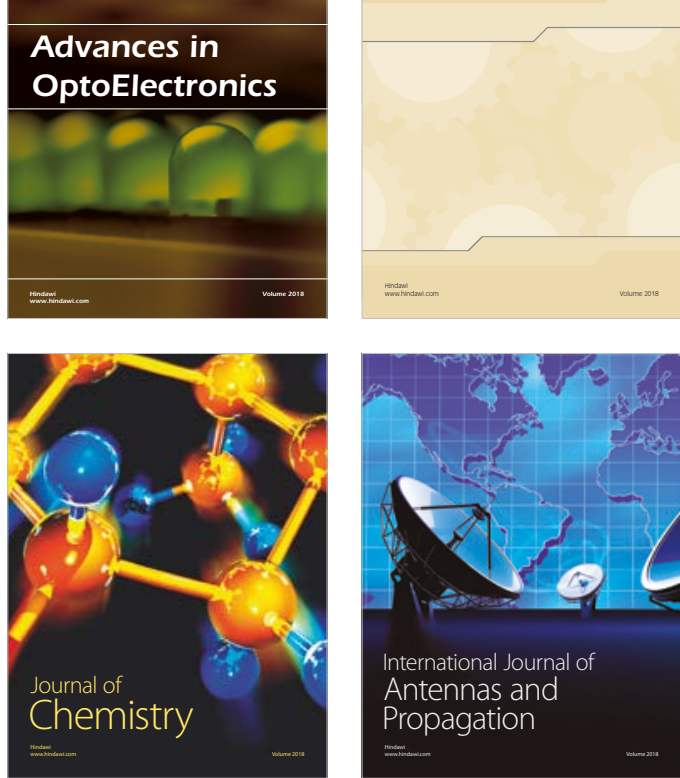

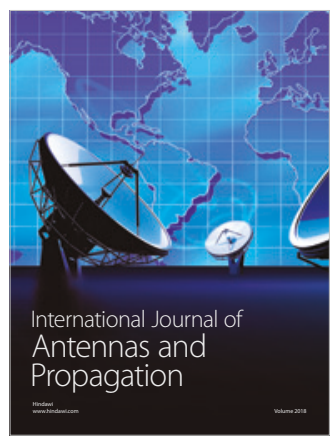

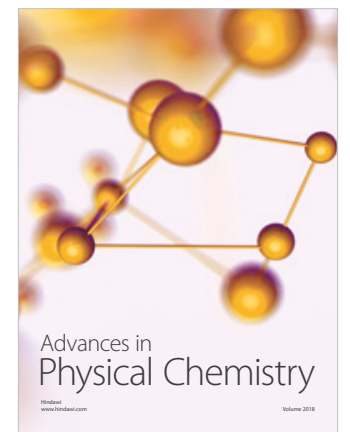

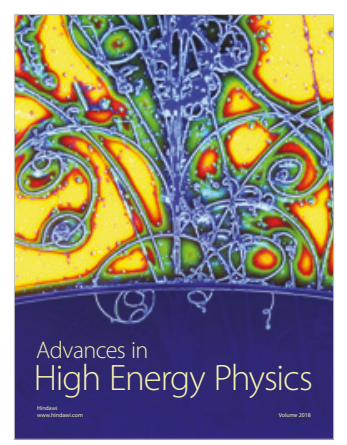

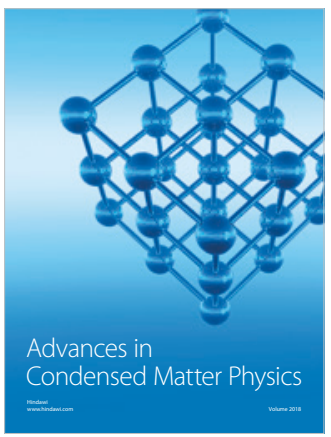

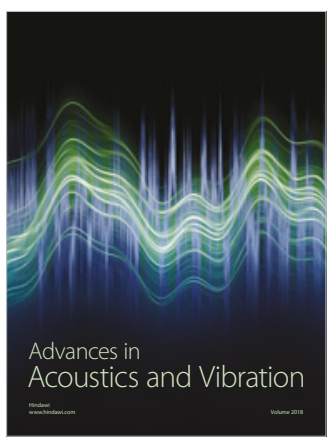

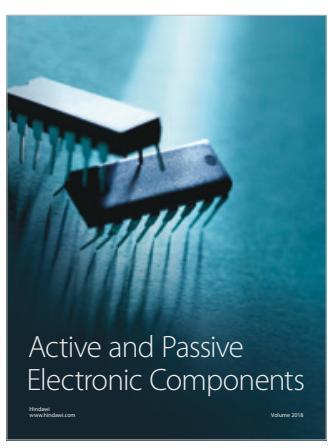
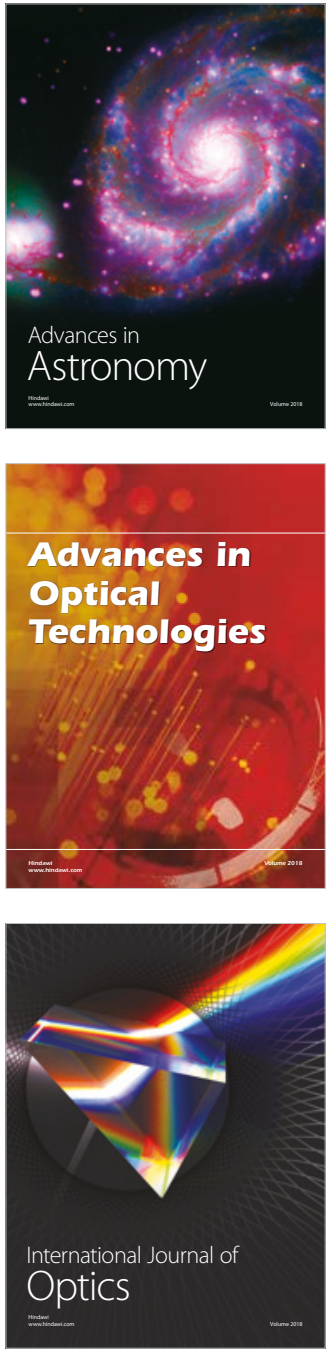